\title{
De la sujétion à la citoyenneté itinéraire du concept de ra iya
}

Azzeddine Allam

\begin{abstract}
Résumé
Dans la pensée politique marocaine traditionnelle, le terme ra'iya revient de façon récurrente pour désigner les gouvernés et établir leurs devoirs vis-à-vis du sultan. Objet du pouvoir sultanien, ra'iya doit en premier lieu s'abstenir, comme l'explicite toute la littérature politique sultanienne de toute action politique. L'utilisation d'un tel concept recèle, bien entendu, une conception de l'espace politique, une vision du rapport État/société. Dans toute la littérature politique sultanienne, il n'est jamais question de « peuple », « nation », « citoyen » ... concepts relevant d'un autre contexte socio-politique. Cette approche traditionnelle a dominé la pensée politique marocaine jusqu'au moins la fin du 19e siècle. Elle se situe aux antipodes de la pensée grecque qui conçoit «le citoyen» comme élément de base de la cité-État. En effet, l'esprit de cette approche exclut catégoriquement les concepts politiques grecs, lesquels concepts seront revifiés, revitalisés, avec l'Europe de la Renaissance, et diffusés après avoir été revigorisés, et somment par conséquent les penseurs marocains de se positionner vis-à-vis de cette nouvelle conception du politique. Dans ce sens on est amené à se poser la question lorsqu'un éminent penseur comme Abdallah Laroui constate avec étonnement qu'au cours du 19e siècle, les penseurs marocains, n'ont rien ajouté aux classiques, et que face à des problèmes cruciaux, ils ne trouvent rien de mieux que de rappeler la célébre « loi circulaire » attribuée à Aristote et abondamment citée par leurs prédecesseurs. En effet l'esquisse d'une effervescence conceptuelle est perceptible dés la fin du 19e siècle. Cette effervescence s'accentuera avec les premières élaborations, constitutionnelles (1906/ 1908), et prendra une autre dimension avec les écrits de quelques auteurs marocains réformistes et salafites. Cette contribution consiste à s'interroger sur la
\end{abstract}

* Université Hassan II-Mohammadia, Maroc 
manière adoptée par la pensée politique marocaine afin de composer avec un nouvel appareil conceptuel. L'a-t-on rejeté partiellement ou totalement ? A-ton essayé de l'endogéneiser? S'agit-il en dernière analyse de cohabitation conceptuelle ou d'un phagocyte entraînant l'élimination des concepts «indigènes» •? Bref, il est question dans cette étude de suivre et de vérifier l'itinéraire d'un concept : ra'iya (sujets).

\section{Abstract}

In traditional Moroccan political thought, the word ra'iya is commonly used to describe those who are governed and also to define their obligations towards the sultan. Ra'iya, as object of the sultan's power, must first and foremost refrain from any political action, as written in the political literature. of the sultanate. The use of such a concept is in consonance with a given conception of political space; it is a specific vision of the relationship between the state and society. In the whole sultanian political literature, there is no mention of the words 'people' 'nation', citizen', which are concepts belonging to another social and political context. This traditional approach dominated Moroccan political thought up to at least the end of the nineteenth century. It is in contrast to the Greek thought which sees the citizen as the foundation of the city state. Indeed, the spirit of this approach excludes the Greek political concepts, which were then revived and disseminated by Renaissance Europe; Moroccan thinkers seek to position themselves vis a vis this new conception of politics. Abdallah Laroui, a great thinker, is surprised that in the nineteenth century, Moroccan thinkers did not contribute any thing new to the classics, contenting themselves with quoting Aristotle's famous 'circular law' to address critical problems. These changes were reinforced by the first constitutional acts (1906/1908), and also with the writings of a few Moroccan reformist and Salafist authors. This paper analyses the strategy followed by Moroccan political thinkers, in reconciling themselves with a new conceptual system. Was it partially or totally rejected? Was there an attempt at endogenisation? Is it about accommodating these concepts or of swallowing up 'indigenous' concepts? In a nutshell, the paper follows the evolution of the concept of ra'iya (subjects).

La présente étude s'organise autour de deux concepts: « sujet » et « citoyen ». Deux difficultés majeures tracent les limites de notre approche :

- Il s'agit de concepts désignant, dans l'un et l'autre cas, une réalité marginalisée, dont le référent fait, le plus souvent, l'objet d'une mise à l'écart indissolublement politique et intellectuelle. D'une part, ar-ra 'iya - troupeau, et, par extension, l'ensemble des assujettis à l'autorité absolue du berger-sultan -, concept clé pour l'analyse du champ politique arabomusulman (et marocain), n'a que faiblement retenu l'attention des 
chercheurs. Il suffit, pour s'en convaincre, de se reporter à la masse considérable d'ouvrages consacrés à l'histoire politique marocaine, et plus largement maghrébine, qui se focalisent sur l'État et les institutions, la personne du sultan et ses actes, les guerres ou la diplomatie, et ignorent totalement ce que l'on désignerait aujourd'hui comme histoire sociale. Il n'est pas du ressort de la présente étude d'interroger les causes de ce désintérêt, dont on peut néanmoins souligner qu'il traduit la difficulté persistante de la société en même temps que des individus et des groupes qui la composent à exister politiquement et « théoriquement », pourraiton dire, face à l'éminence du pouvoir sultanien - même quand celui-ci est le plus vivement contesté ${ }^{1}$. D'autre part, le terme «citoyen » (muwatin) ou «citoyenneté »(muwatana), très récent dans notre vocabulaire politique, reste vague, ambigu et polysémique, renvoyant à des valeurs à la fois politiques, sociales et culturelles. La surcharge de ce terme et l'effervescence qu'il suscite aujourd'hui dans les esprits traduisent le fait que la citoyenneté, au sens large du terme, n'est pas totalement acquise et reste par conséquent un idéal à poursuivre et à concrétiser.

_ $\quad$ La deuxième difficulté relève de l'objet même de la présente étude: « De la sujétion à la citoyenneté ». Il serait outrecuidant et pour le moins prématuré, en l'absence d'analyses fines de prétendre être en mesure de détecter les ruptures très lentes et les transformations parfois invisibles qui se sont opérées et sont toujours à l'œuvre entre deux concepts antagonistes que leur charge sémantique et politique situe aux antipodes l'un de l'autre. En tout état de cause, nous ne prétendons pas livrer ici une étude minutieuse relatant le passage, manifestement toujours inachevé, de la sujétion traditionnelle à la citoyenneté moderne - tâche que seul un ensemble d'études multidisciplinaires pourraient rendre possible. Plutôt, nous nous limiterons, dans un premier temps, à envisager le concept de $r a$ 'iya tel qu'il a été élaboré par les penseurs marocains classiques, pour tenter de suivre, dans une deuxième partie, son éclipse progressive au profit d'autres termes qui débouchent, plus ou moins, sur l'émergence d'une « citoyenneté », au sens moderne de ce terme.

\section{Ar-ra'iya dans la pensée politique marocaine traditionnelle}

Dans toute la littérature politique sultanienne marocaine ${ }^{2}$, depuis le Kitàb as-siyàsa (le livre de la politique) composé par Abû Bakr AI-Murâdi al-Hadrami au XIe siècle de l'ère chrétienne (IVe siècle de l'Hégire), jusqu'à At-tàj wal-iklil (le Livre de la couronne) d'Abü al-Qâsim AzZayyâni (mort en 1830), le terme ar-ra 'iya revient de façon récurrente pour désigner les gouvernés et établir leurs devoirs vis-à-vis des sultans. 
Objet du pouvoir sultanien, ar-ra 'iya doit, en premier lieu, manifester son allégeance et notamment, comme l'explicite Ibn Abi ar-Rabi', «s'abstenir de toute action politique $»^{3}$. L'utilisation presque exclusive de ce terme pour désigner les gouvernés traduit une conception de l'espace politique et une vision du rapport État/société basées sur l'appropriation par le pasteur de son troupeau et le devoir qui lui incombe d'orienter celui-ci ${ }^{4}$.

Cette littérature est unanime à considérer ar-ra'iya comme l'assise de tout pouvoir: Abü Bakr At-Tartushi (XIe siècle) estime ainsi que l'entretien de celle-ci est de nature à assurer la stabilité politique plus efficacement que la mise sur pied d'une armée puissante ${ }^{5}$. AI-Murâdi (XIe siècle), Ibn Rad wan (XIVe siècle) et Ibn Al-Azraq (XV siècle) s'accordent à considérer qu' ar-ra'iya demeure la seule provenance des ressources indispensables pour l'entretien de l'armée qui est elle-même nécessaire pour assurer la continuité du pourvoir sultanien ${ }^{6}$. Sans multiplier les références, on peut souligner d'emblée un aspect paradoxal de ces écrits. À côté d'affirmations qui confèrent aux « sujets » un intérêt particulier, les auteurs sultaniens développent à leur égard une vision à la fois « passive » et «négative ». Un certain nombre de métaphores véhiculées par cette littérature en témoignent: AI-Mawardi conçoit ar-ra 'iya comme un « orphelin » ayant besoin d'un tuteur pour le protéger ${ }^{7}$; At-Tartushi y voit un « corps » pâle et affaibli à défaut. d'être habité par l'âme sultanienne, une obscurité nécessitant « le flambeau des rois », une terre sèche implorant l'eau sultanienne ${ }^{8}$. Ibn Radwan, Abou Hamou Az-Ziyyini, sultan de Tlemcen, et Ibn Al-Azraq y voient, pour leur part, un «malade » ayant besoin d'un médecin, en l'occurrence le sultan? Toutes ces images renvoient à la conviction unanime de ces auteurs qui considèrent que « les sujets » livrés à eux-mêmes sont voués à la perdition et au désordre meurtrier. Ils ne peuvent exister, ni coexister, sans l'assistance du "wazi"' (puissance coercitive et incitative) sultanien. Plus encore, ces auteurs ne se contentent pas de faire l'apologie du pouvoir et de sa nécessité, mais ils recommandent aux sultans, étant donnée la nature malveillante et maléfique des « sujets », de se montrer fermes et vigilants à leur endroit. Il suffit, à cet égard, de rappeler les différentes qualifications qui fleurissent sous la plume de ces auteurs: al-'amma (le commun), ad-dahma (la foule), al-ghawgha' (la masse, mob en anglais), as-süqa (la multitude), al-awbash (la lie), ar-ru'at (le troupeau), aljarad (les sauterelles) ; ces désignations veulent bien dire ce qu'elles veulent dire. 
Trois éléments au moins corroborent cet aspect passif et négatif imputé aux "sujets".

\section{Les techniques de l'emprise sultanienne}

Le terme ra'iya n'est, on l'a souligné, jamais traité en lui-même, de manière indépendante, mais toujours en rapport avec le pouvoir du sultan, comme cela apparaît, en particulier à travers les conseils politiques prodigués aux sultans par les auteurs de cette littérature et dans les règles de conduite auxquelles ils lui recommandent de se tenir. De l'ensemble de cette éthique politique, on peut déduire deux techniques principales ${ }^{10}$ qui servent de base à tout comportement sultanien : la technique de la séduction, at-targhib, la technique de l'intimidation, at-tarhib.

La première technique vise à conquérir « les cœurs » des sujets, en créant une atmosphère d'amour et de confiance, comme condition préalable d'un règne stable et prolongé. Elle assure au sultan l'image d'un être identifiable à un père, bienveillant, beau, généreux, compréhensif et miséricordieux. Le sultan mobilise, grâce à cette technique, ce que Elias Canetti désigne comme «l'économie de l'Éthique», une logique où derrière chaque «vertu » sultanienne se cache une contrepartie politique: il donne d'une main pour reprendre de l'autre, échange sa générosité ou exerce « la puissance de son pardon » pour recevoir soumission et allégeance. ${ }^{11}$

Toutefois, cette technique a ses limites. Elle ne peut à elle seule, assurer la pérennité de l'ordre établi, Pour ces auteurs, la grandeur, ou la vénération mêlée de crainte (al-hayba, ar-rahba), demeurent le moyen le plus sûr de se maintenir au pouvoir et de protéger celui-ci contre tous les prétendants. Cette technique correspond à la nature et à la logique même du pouvoir sultanien. Grâce à ses effets les sujets sont intimidés et nourrissent des sentiments de faiblesse et d'infériorité devant la machine sultanienne prête à écraser tout ce qui se mettrait en travers de son chemin. Les manifestations de cette technique épousent toutes les formes à travers lesquelles se matérialise la visibilité du pouvoir: étiquette de la cour sultanienne, manifestation des hiérarchies ${ }^{12}$, et jusqu' aux sorties publiques des sultans. ${ }^{13}$

\section{Les espèces d'ar-ra'iya}

D'un point de vue méthodologique, faut-il pour autant écarter toute interprétation sociologique « positiviste »? En effet, on est en présence de classifications fondamentalement «normatives »- les nota- 
bles généreux (an-nubala', al-akram), les gens moyens (al-awsat), les vilains (as-safal ${ }^{14}$ - ou encore de classifications vagues sans consistance objective: al-amma (le peuple), al-khassa (l'élite), khassat al-khassa (l'élite de l'élite)... . ${ }^{15}$ Et même lorsque les auteurs s'essayent à des classifications « socio-professionnelles »-shurafa', saints, fuqaha', commerçants, notables... ${ }^{16}$, c'est strictement dans un souci « subjectif », à savoir, celui d'évaluer les positions de chaque catégorie par rapport au sultan. Ce qui préoccupe les auteurs sultaniens, dans toutes ces classifications n'est autre que la «valeur d'usage » que seul, le sultan est en mesure de consommer.

\section{Droits et devoirs d'ar-ra'iya}

At-Tartushi, dans l'intitulé. du chapitre 40 de son traité, se pose la question de savoir ce que les sujets doivent faire au cas où le roi serait injuste. La réponse est argumentée, tout au long de son texte, par une série de citations -versets coraniques, Hadith-s, récits, adages, dictons..., qui s'organisent autour de deux points: la proscription (au sens religieux du terme) de toute révolte (khuruj) au nom de la sauvegarde de l'unité de la communauté, et l'exaltation de la vertu de patience devant les actes injustes pour les gouvernés. Mieux encore, le bien des sujets consiste « à s'abstenir de toute action, parole ou comportement, se rapportant aux affaires de l'État». Affirmant l'aspect à la fois politique et religieux du devoir de soumission, les auteurs sultaniens implorent les sultans de faire prévaloir les « droits de la sujétion » : justice, sécurité, application du shar'. etc. Cette série de $"$ droits $»^{17}$ nous suggère deux remarques. D'une part, il serait proprement ahistorique de nous livrer à quelque forme d'analogie que ce soit entre les droits de la « citoyenneté » et ceux de la «sujétion»: la dimension à la fois positive et institutionnalisée des premiers est fondamentalement incompatible avec la dimension «subjective » des seconds; la citoyenneté ne procède de rien d'autre que d'elle-même, ou du moins de l'institution qui l'énonce comme abstraction, tandis que la sujétion concrète est intimement liée au caractère personnel, individuel des sultans. D'autre part, comme l' a montré Ibn Khaldun, il est toujours de l' intérêt du sultan de se comporter conformément à la justice ('adl); cela favorise la longévité du pouvoir, alors que l'injustice conduit nécessairement à sa destruction. Cette 
approche sultanienne, qui ne perçoit les sujets qu'à travers le prisme du pouvoir du sultan, a dominé la pensée politique marocaine au moins jusqu'à la fin du XIXe siècle. Les fondements de cette approche excluent catégoriquement les concepts politiques grecs, lesquels seront empruntés à l'Europe des Lumières et très lentement diffusés après avoir été revivifiés et mettent en demeure les penseurs marocains de se positionner vis-à-vis de cette nouvelle conception politique.

\section{Ine transition politique?}

Entre le dernier tiers du XIXe et le début du XXe siècle, on peut identifier trois types de documents susceptibles de nous éclairer sur l'itinéraire du concept de ra 'iya : la littérature politique makhzénienne, les chroniques de voyages et les premiers projets constitutionnels.

S'il est vrai que la situation globale du Maroc au XIXe siècle et les événements qui s'ensuivirent - occupation de l'Algérie, défaite d'Isly, guerre de Tétouan, menaces d'occupation, troubles sociaux, déliquescence de l'appareil étatique... - ont poussé les réformateurs marocains à centrer leurs discours sur l'État, il serait néanmoins déplacé d'attendre des réponses à des questions qu'ils n'ont pas posées. Néanmoins leurs écrits nous permettent de formuler quelques remarques :

- $\quad$ La littérature makhzénienne du XIXe siècle témoigne, comme l'a montré A. Laroui à propos d'Akansüs, d'un « irréalisme » surprenant, comme si rien ne s'était passé. Elle ne fait que reproduire exactement ce qu'aladab as-sultani avait déjà mâché et remâché. Ces textes sont pleins de « réminiscences » et n'ajoutent rien aux « classiques » du genre. Les chapitres en sont sans grande originalité: face à un problème, on ne trouve pas d'analyses «théoriques», mais seulement un exposé de cas de "figure" ou d"'espèce". Comme par le passé, cette littérature ne constitue pas un «moyen d'action » mais plutôt un simple «signe de distinction $\gg^{18}$. Cependant, pourquoi faudrait-il s'étonner de trouver au cœur du XIXe siècle marocain la même conception de la sujétion déjà établie par un Tartushi ou un Ibn Radwan ? En fait, le seul aspect qui distingue cette littérature makhzéniene du XIXe siècle finissant de ce qui l'a précédée, c'est, très fortement, cette «anxiété pathologique » qui travaille ces textes et ne cesse d'affleurer face à une Europe montante et menaçante qui bouscule l'ordre social culturel et politique du sultanat.

- $\quad$ À l'inverse de la réticence très explicite de ceux qui ont vu l'Europe venir jusque chez eux, les chroniques des quelques voyageurs marocains qui ont pu voir l'« Europe » de l'intérieur témoignent, globalement, d'une 
certaine fascination à son endroit eu égard à sa puissance, à son organisation et à ce que l'on s'accordera à désigner comme sa « civilisation » tamaddun, hadara... - forte, non sans que ne s'expriment de fortes réserves, notamment en matière de religion ou d'éthique. Outre l'admiration qu'il exprime à propos des moyens de communication, et notamment du chemin de fer, de l'architecture des villes et de la propreté des gens, $\mathrm{Mu}$ hammad Ibn' Abd Allah As-saffar découvre l'inconcevable liberté dont jouit ce que l'on désignerait aujourd'hui comme la «société civile »: «Parmi les lois que leur sultan a promulguées figure l'interdiction d'empêcher toute personne de s'exprimer librement et d'écrire ce que lui dicte sa conscience ». Mieux encore, il rend compte de la révolution qui renverse Charles X en expliquant que celui-ci voulait censurer la presse. D'autre part, il évoque l'institution parlementaire, le mode électif de sa désignation et ses attributions, identifiant les députés comme les "délégués des sujets" dont la mission est de contrôler le gouvernement ${ }^{19}$. Soulignons, chez As-Saffar et quelques autres ${ }^{20}$, cette persistance de la terminologie politique sultanienne sultan, ra'iya, bayt al-mal, pour rendre compte d'un monde perçu comme tout autre. Comment passe-t-on de « citoyen-électeur » à ra'iya - ou l'inverse -? Problème de langage ou problème politique ?

Les premiers projets « constitutionnels » qu'a connus le Maroc, au début de ce siècle, témoignent d'une certaine effervescence conceptuelle ${ }^{21}$. Ils constatent la déliquescence de la bureaucratie sultanienne et plaident pour une réforme politique et l'instauration d'un État « juste», ce que l'on désignerait aujourd'hui comme un «État de droit». On continue de parler d'ar-ra'iya dans ces textes constitutionnels, mais force est de constater l'émergence d'autres concepts qui reflètent cette volonté de réformer le champ politique. Le projet de 'Ali Zniber, tout en utilisant le terme ra'iya dans un sens «passif », en tant qu'objet du pouvoir $^{22}$ (art. II), témoigne de l'émergence d'une nouvelle terminologie $^{23}$. Il n'est pas moins significatif de constater que dans le projet de constitution publié par Lisan al-Maghrib en $1908^{24}$ il n'est pas fait mention d'ar-ra'iya. Il est vrai qu'il n'y est pas non plus question de «citoyen », même si on peut soutenir l'idée qu'une telle notion était implicitement présente dans l'esprit de cette constitution qui reste l'une des premières tentatives de modernisation du champ politique marocain. En tout état de cause, l'éclipse d'ar-ra'iya ouvre la voie à une série de notions: «individu » (fard), « marocain » (maghribi), « électeur » $($ nakhib $)$, « peuple » (sha'b), « habitants » (sukkan)... De tous ces termes, c'est celui d"'Umma"- « communauté », plutôt que «nation »qui revient le plus souvent tout au long des articles de ce projet. Pris 
dans son ensemble, il constitue bel et bien un premier pas vers la citoyenneté: al-umma est au fondement de la représentativité (at-tamthlliya) (art. 46) ; elle incarne l'intérêt public "as-salih al 'amm" (art. 19) ; toute trahison à son égard est sévèrement punie (art. 40); elle a des droits (art 54) ; elle est assujettie à l'impôt (art 19) ; elle a le droit de poursuivre n'importe quel fonctionnaire de l'État (art 34).

S'agit-il pour autant vraiment d'une rupture avec la pensée politique traditionnelle? La réponse est forcément négative dans la mesure où les deux terminologies, traditionnelle et moderne - voisinent et cohabitent tant bien que mal dans l'esprit de ces réformateurs. Cette cohabitation, souvent artificielle et sélective, est clairement perçue chez d'autres réformateurs marocains comme 'Abd ar-Rahman Ibn Zidan (m. en 1946) ou Muhammad Ibn al-Hasan Al-Hajwi (m. en 1956). Ainsi la soumission des «sujets » est-elle conditionnée par la sauvegarde des intérêts de la nation. $A l-a d l$, la justice, avec son fondement religieux, est identifiée aux lois « positivistes »; quant à ceux qui délient et lient ( $\mathrm{Ahl}$ alhall wa al-'aqd), ils sont identifiés aux institutions parlementaires,.. ${ }^{25}$

En effet, il est frappant de retrouver la même confusion conceptuelle chez un penseur comme 'Allal al-Fassi (m en 1975) qui, lui non plus n'a pas pu se détacher ou du moins se distancier de l'arsenal conceptuel traditionnel qui a pesé lourd dans la pensée politique marocaine ${ }^{26}$.

'Allal al-Fassi, grand personnage, homme politique, faqih réformateur salafite, poète... s'est servi de matériaux disparates pour élaborer son projet. Il est vrai qu'aux yeux de' Allal, qui a développé sa pensée politique contre la pensée sultanienne ${ }^{27}$, les gouvernés constituent dorénavant un « peuple », une « nation », un « ensemble de citoyens ». Pour lui, il ne s'agit plus d'un sultan et d'une ra'iya mais d'un « roi » et d'un « peuple $»^{28}$, Toutefois faut-il signaler que sa pensée n'est pas aussi simple qu'elle le paraît, et que, loin de constituer une théorie cohérente et systématique, les idées politiques et sociales éparpillées dans son œuvre répondent en fait aux exigences du moment historique.

La terminologie moderne dont se sert 'Allal témoigne-t-elle d'une rupture avec la terminologie traditionnelle? Est-ce un hasard qu'il défende la liberté et notamment la liberté politique dans un ouvrage intitulé Maqasid ash-sharia? En effet 'Allal al-Fassi projette sur le passé un avenir probable, Il s'efforce de trouver dans son héritage historique les bases de la modernité, en particulier le principe de «la participation politique », Ainsi la « constitution », de Médine, la Bay'a des premiers califes, le «conseil » créé par le calife 'Abd Al- 'Aziz, ou encore 
l'institution traditionnelle de la jmaa' constituent des bases possibles de la participation politique.

La shura devient elle aussi synonyme de « démocratie » et signifie la capacité du citoyen à légiférer selon son ijtihad. Cette confusion conceptuelle, où islam, démocratie et socialisme se fondent, est l'horizon de la pensée politique de 'Allal al-Fassi et l'a empêché de concevoir l'État comme valeur «autonome » et indépendante en dehors des valeurs religieuses et morales ${ }^{29}$

En effet, la prédominance de l'instant politique (indépendance du pays, construction de l'État ...) qui a empêché toute préoccupation théorique d'une citoyenneté « virtuelle » ou du moins en cours de construction, nous incite à ne pas attendre des réponses à des questions que Allal Al Fassi n'a pas posées ou du moins des questions qui n'étaient pas à l'ordre du jour de ses réflexions.

Enfin, qu'en est-il aujourd'hui, sommes-nous des sujets ou des citoyens? Il est significatif à cet égard que le thème de la citoyenneté avec toutes les questions qui en découlent se trouve d'une manière explicite ou implicite au centre des préoccupations des Marocains. Il est quasiment présent dans les discours de l'État, des partis politiques, des syndicats, des organisations et associations civiles.

Le milieu intellectuel marocain, pour sa part, a connu au cours de ces dernières années, des débats et de réflexions autour du concept de la « société civile ». Face à l'esprit manichéen marquant la plupart de ces débats (affirmer ou nier l'existence de cette société civile), on a le droit de s'interroger sur la pertinence d'utiliser un autre terme émanant de notre fond culturel, à savoir celui de Arra'iya, mot arabe qui, curieusement, n'a pas de singulier.

\section{Notes}

l Mohamed Arkoun remarque, en ce sens, que l'étude sociologique de la société arabo-musulmane apparaît comme très difficile, sinon impossible, dans la mesure où la quasi-totalité des « ressources » qui nous sont parvenues écartent, sous-estiment et dénigrent ce que cette littérature désigne comme al-'amma litt. le commun des mortels et, par extension, la «plèbe » ou le « peuple » par opposition à al-khassa, l'élite. Voir Arkoun 1990 : 116 . Pour ce qui concerne le Maroc, des historiens comme Abdallah Laroui, Mohamed Zniber et d'autres ont insisté sur la pertinence de faire valoir la dimension sociale de notre histoire. Voir aussi Sebti, s. d.; Mezzine 1989; Tahiri 1991.

2 Je me permets de renvoyer le lecteur à Allam 1990. 
Allam : De la sujétion a la citoyenneté itinéraire du concept de ra’iya

3 Ibn Abi ar-Rabi', Sulük al-malikfi tadbir al-mamilik (Des comportements du roi dans l'administration de ses États) 1978 : 138.

4 Étymologiquement, ra'iya signifie le troupeau de moutons ou de brebis. Voir Lissan al-'Arab.

5 At-Tartüshi, Sirij al Mulük (Le flambeau des rois) 1990 : 340.

6 Ibn Radwan, Ash-shuhüb al-limi'afiy-siyisa an-niji'a (Les étoiles brillantes dans les politiques utiles) 1984 : 100 ; Ibn Al-Azraq, Bada'i' as-sulkfi tabi'i' al-mulk (Les conduites les plus éclatantes du point de vue de la nature des royaumes) 1977.

7 AI-Mawardi, Ta bl7 an-nazarfi akhliq al-malik wa siyisat al-mulk (De l'éthique du roi et la politique du royaume) : 214 .

8 At-Tartushi, op. cit. Voir, en particulier, le chapitre 9.

9 Abü Hamü Az-Ziyyini, Wisitat as-sulükfi siyisit al mulük (Des conduites médianes dans les politiques des rois), manuscrit n 1298, Bibliothèque générale de Rabat, p. 34; Ibn AI-Azraq, op. cit.

10 L'utilisation du terme « technique » trouve ici sa justification dans le fait que les écrits politiques dits «sultaniens », al-adab as-sultini, étaient destinés exclusivement au sultan lui-même, pour qui il s'agissait d'exposer les règles à suivre tant en matière de comportement personnel « privé » (façon de se vêtir, de manger, comportement avec les femmes, palais...) que «public » (choix des ministres, secrétaires et gouverneurs... entretien de l'armée, rapports avec les sujets.etc.). Voir à ce sujet Allam 1990.

11 Canetti $1966: 317-318$.

12 Voir par exemple: Ibn Radwan, op. cit., chapitre 5 ; At-Tartushi, op. cit. Chapitre 25 ; Ibo Al-Azraq, chapitre 15.

13 Al-Muradi, op. cit., chapitre 8 et 9.

14 Cette classification tripartite est récurrente dans toute la littérature politique sultanienne. Voir à titre d'exemple AI-Muaidi, op. cit. : 13 ; Ibn AI-Khatib, Maqama as-siyisa (la séance de la politique). Rabat, s.d. : 123 ; Ibn Tabataba, Alfakhrifl al-adab as-sullani (Morceaux choisis de la littérature sultanienne), textes publiés par Kamil Sha'bina, Beyrouth, Dar Beyrouth, 1980 : 41.

15 Voir à ce propos: Encyclopédie de l'Islam (nouvelle édition), t. IV : 1128.

16 Par exemple, Ibn Abi al-Rabi', op. cit., p. 147; Abü Hamü, op. cit., p. 79; Ibn AlAzraq,. op. cit., t. I, chap. 18.

17 Voir, à titre d'exemple, Al-Mawardi, op. cit., p. 214.

18 Laroui 1993 (réédition) : 222-23.

19 Voir à ce propos, ' Abd Il-llah Belqaziz, Al-khitab al-islahi fil-Maghrib (Le discours réformateur au Maroc) 1997 : 95-100.

20 Par exemple, Idris Ibn Idris Al-' Amrawi, Tuhfat al-malik al-'aziz bi-mamlakat Bariz.

21 Voir Muhammad al-Manuni, Mazahir yaqazat al-Maghrib al-hadith (Les manifestations de l'éveil du Maroc moderne) 1985, t. 2 : 399 et suiv.; A. Belqaziz, op. cit : 187-94.

22 La bay'a de Fès comporte les termes ra 'iya et umma dans deux sens opposés. Le premier représente $a r$-ra 'iya en tant qu'être passif et objet du pouvoir sultanien, le deuxième confère à la umma un rôle « positif » : le sultan ne peut conclure 
un accord avec un État étranger qu'après en avoir informé al-umma. Celle-ci est devenue un acteur qui rejette les clauses d'Algésiras.

23 Notons, entre autres, la présence de notions telles qu' «élection » (art. I), «contrôle du pouvoir exécutif » (art.8), "égalité » (art. 9).

24 Voir par exemple Abd al-Karim Ghallab, At-Tatawwur ad-dusturi wal-niyabi filMaghrib (L'évolution constitutionnelle et parlementaire du Maroc), n. d., s. n. é.

25 Voir, à titre d'exemple Benyoussef 1994.

26 Voir à ce propos Laroui 1981.

27 Voir Meski 1996-1997.

28 Ibid., p. 114-130.

29 Voir à ce propos Laroui 1981.

\section{Réiérences bibliographiques}

Abd al-Karim Ghallab, At-Tatawwur ad-dusturi wal-niyabi fil-Maghrib (L'évolution constitutionnelle et parlementaire du Maroc), n. d., s. n. é.

' Abd Il-1!ah Belqaziz, Al-khitab al-islahi fil-Maghrib (Le discours réformateur au Maroc), 1997, Beyrouth : Dar al-Muntakhab : 95-100.

Abü Hamü Az-Ziyyini, Wisitat as-sulükfi siyisit al mulük (Des conduites médianes dans les politiques des rois), manuscrit $\mathrm{n}^{\circ} 1298$, Bibliothèque générale de Rabat, p. 34;

Abü Hamü, op. cit., p. 79;

Allam, Azzedine, 1990, Pouvoir et politique dans la littérature sultanienne. Casablanca : Afrique-Orient.

Al-Manuni, Muhammad, 1985, Mazahir yaqazat al-Maghrib al-hadith (Les manifestations de l'éveil du Maroc moderne), Beyrouth : Association marocaine des Auteurs, t. 2 : 399 et suiv.;

AI-Mawardi, Ta -bl7 an-nazarfi akhliq al-malik wa siyisat al-mulk (De l'éthique du roi et la politique du royaume), texte annoté par R. Es-Sayyid, Beyrouth : Dar al-'ulüm al-'arabiya : 214.

Arkoun, Mohamed, 1990, Islam, éthique et politique (en arabe), Beyrouth : Markaz aI- inmâ I al-qawmi : 116.

At-Tarlüshi, Sirij al Mulük (Le flambeau des rois), 1990, texte publié et annoté par Ja'far al-Bayati. Londres : Riyad ar-Rayyis : 340.

Benyoussef, Najia, 1994, «L'œuvre politique d'ibn Zidan », in Abhath, n 31/32, Rabat.

Canetti, Elias, 1966, Masse et puissance, Paris : Gallimard : 317-318.

Encyclopédie de l'Islam (nouvelle édition), t. IV : 1128.

Ibn Abi ar-Rabi', Sulük al-malikfi tadbir al-mamilik (Des comportements du roi dans l'administration de ses États), 1978, texte publié et annoté par Naji At-Takliti, Beyrouth : Dar 'Uwaydat: 138.

Ibn Al-Azraq, Bada'i' as-sulkfi tabi'i' al-mulk (Les conduites les plus éclatantes du point de vue de la nature des royaumes), 1977, texte publié par Simi An-Nashshir, Ministère de la Culture, Bagdad.

Ibn AI-Khatib, Maqama as-siyisa (la séance de la politique), s.d., Rabat : 123. 
Allam : De la sujétion a la citoyenneté itinéraire du concept de ra’iya

Ibn Radwan, Ash-shuhüb al-limi'afiy-siyisa an-niji'a (Les étoiles brillantes dans les politiques utiles) 1984, texte publié par Simi An-Nashshir, Casablanca : Dar aththaqifa.

Ibn Tabataba, Al-fakhrifl al-adab as-sullani (Morceaux choisis de la littérature sultanienne), 1980, textes publiés par Kamil Sha'bina, Beyrouth : Dar Beyrouth.

Laroui, A.,1981, Mafhum ad-dawla (le concept d'État), ch. V, Casablanca : Centre culturel arabe.

Laroui, A., 1993 (réédition), Les origines sociales et culturelles du nationalisme marocain (1830-1912), Casablanca : Centre culturel arabe.

Meski, Mohamed, 1996-1997, Le discours politique d'Allal El Fassi, mémoire de DES, Faculté de Droit Casablanca.

Mezzine, Mohamed, 1989, «An-nawizil al-fiqhiya et l'écriture de l'histoire », in La recherche dans l'histoire du Maroc. Bilan et évaluation, Rabat : Publications de la Faculté des Lettres de l'Université Mohamed V.

Sebti, Abdelahad, s.d., L'histoire sociale et la problématique de la méthode, Rabat: $\mathrm{Pu}-$ blications de la Faculté des Lettres de l'Université Mohamed V.

Tahiri, Ahmed, 1991, «AI-'âmma dans la société islamique médiévale », in Aspects de l'histoire sociale des pays méditerranéens au moyen âge, Meknès : Publications de la Faculté des Lettres de Meknès. 
